\title{
COEFFICIENTS OF BLOCH FUNCTIONS AND NORMAL FUNCTIONS
}

\author{
J. S. HWANG and PETER LAPPAN
}

Abstract. We give a number of sufficient conditions for an analytic function $f(z)=\Sigma\left(a_{n}+b_{n}\right) z^{n}$ to be a normal function. In particular, we show that if $\left\{a_{n}\right\}$ is a decreasing sequence of non-negative real numbers, and if $\left\{b_{n}\right\}$ is a sequence which satisfies both $b_{n}=O(1 / n)$ and $\sum_{n=p}^{\infty}\left|b_{n}-b_{n+1}\right|=$ $O\left(b_{p}\right)$, then $f(z)$ is a normal function. Some of our results deal with Bloch functions.

\section{Introduction}

Let $D$ denote the unit disc $\{z:|z|<1\}$. A function $f(z)$ meromorphic in $D$ is called a normal function if

$$
\sup \left\{\left(1-|z|^{2}\right) f^{\#}(z):|z|<1\right\}<\infty,
$$

where $f^{\#}(z)=\left|f^{\prime}(z)\right| /\left(1+|f(z)|^{2}\right)$. It is well known that a meromorphic function which omits 3 values is a normal function, and we will use this fact in what follows.

A function $f(z)$ analytic in $D$ is called a Bloch function if

$$
\sup \left\{\left(1-|z|^{2}\right)\left|f^{\prime}(z)\right|:|z|<1\right\}<\infty \text {. }
$$

It is easily seen that a Bloch function is a normal function.

In [1] and [2], the second author studied conditions on the coefficients $\left\{a_{n}\right\}$ which are sufficient to make the function $f(z)=\sum a_{n} z^{n}$ a normal function. In particular, the following two results were proved in [1].

Theorem A. If $\left\{a_{n}\right\}$ is a bounded monotone sequence of real numbers, then $f(z)=\sum a_{n} z^{n}$ is a normal function.

Theorem B. If $\left\{a_{n}\right\}$ is a sequence of complex numbers such that $\sum\left|a_{n}-a_{n+1}\right|<\infty$ and $\lim _{n \rightarrow \infty} a_{n}=0$, then $f(z)=\sum a_{n} z^{n}$ is a normal function.

Here, we investigate a number of results in the spirit of Theorems A and B, with some consideration of Bloch functions. Essentially, our investigation asks what

1985 Mathematics Subject Classification. Primary 30D45. Key words and phrases: Normal function, Bloch function, nearly monotone sequence, naturally convex sequence. 
happens when a function whose sequence of coefficients satisfying the hypotheses of Theorem $A$ is added to a function whose sequence of coefficients satisfies a condition like the hypothesis of Theorem B. In Section 2, we give a number of preliminary results. Some of these results deal with Bloch functions, and some deal with the behavior of the real and imaginary parts of analytic functions with certain types of coefficient sequences. Our main results appear in Section 3. Some examples are given in Section 4.

\section{Preliminary results}

We begin with some results about Bloch functions.

Lemma 1. If $a_{n}=O(1 / n)$, then $f(z)=\sum a_{n} z^{n}$ is a Bloch function.

Proof. If $a_{n}=O(1 / n)$, then

$$
\left|f^{\prime}(z)\right| \leqq \sum O(1)|z|^{n}=O(1) /(1-|z|),
$$

which means that $f(z)$ is a Bloch function.

Corollary. If $f(z)=\sum a_{n} z^{n}$ is not a normal function, then $\lim \sup _{n \rightarrow \infty} n\left|a_{n}\right|=\infty$.

Theorem 1. If $\left\{a_{n}\right\}$ is a decreasing sequence of positive real numbers, then $f(z)=\sum a_{n} z^{n}$ is a Bloch function if and only if $a_{n}=O(1 / n)$.

Proof. By Lemma 1, if $a_{n}=O(1 / n)$ then $f(z)$ is a Bloch function. Conversely, if $\left\{a_{n}\right\}$ is a decreasing sequence of positive real numbers, then $\sum_{j=1}^{n} j a_{j} \geqq a_{n}\left(\sum_{j=1}^{n} j\right)=$ $[n(n+1) / 2] a_{n}$. However, $\left|f^{\prime}(x)\right|=O(1 /(1-x))$ if and only if $\sum_{j=1}^{n} j a_{j}=O(n)$ (see [4, Section 7.51]). Thus, if $f(z)$ is a Bloch function and $\left\{a_{n}\right\}$ is a decreasing sequence of positive real numbers, then $n(n+1) a_{n} / 2=O(n)$, which means that $a_{n}=O(1 / n)$. This proves the theorem.

Next, we prove a result about Bloch functions with an additional restriction on the coefficients. We say that the sequence $\left\{a_{n}\right\}$ is nearly monotone if $\sum_{j=p}^{\infty}\left|a_{j}-a_{j+1}\right|=$ $O\left(\left|a_{p}\right|\right)$ for each positive integer $p$.

Lemma 2. If $\left\{b_{n}\right\}$ is a nearly monotone sequence of real numbers, and if $f(z)=$ $\sum b_{n} z^{n}$, then $|f(z)|$ is bounded for each $z$ near $e^{i \theta}$ for each $\theta, 0<\theta<2 \pi$. In addition, if $b_{n}=O(1 / n)$ and $b_{n} \geqq 0$ for each $n$, then both $\operatorname{Im} f(z)$ is uniformly bounded for $|z|<1$ and $\operatorname{Re} f(z)$ is bounded below for $|z|<1$.

Proof. Let $g(z)=(1-z) f(z)=b_{0}+\sum_{n=0}^{\infty}\left(b_{n+1}-b_{n}\right) z^{n+1}$. Since $\left\{b_{n}\right\}$ is a nearly monotone sequence, $g(z)$ is continuous and bounded for $|z| \leqq 1$. Thus $f(z)$ is continuous at $e^{i \theta}$ for each $\theta, 0<\theta<2 \pi$, which proves the first part of the lemma.

For proving the rest of the lemma, it is no loss of generality to prove the results for $z=r e^{i \theta}, 1 / 2 \leqq r<1,0 \leqq \theta<2 \pi$. Henceforth, let $b_{n}=O(1 / n), b_{n} \geqq 0$, and $B_{n}=b_{n} r^{n}$ 
for $1 / 2 \leqq r<1$. We claim that for each such $r,\left\{B_{n}\right\}$ is a nearly monotone sequence, since

and so

$$
\begin{aligned}
\left|B_{n}-B_{n+1}\right| & \leqq\left|b_{n} r^{n}-b_{n+1} r^{n}\right|+b_{n+1}\left(r^{n}-r^{n+1}\right) \\
& \leqq\left|b_{n}-b_{n+1}\right| r^{n}+b_{n+1} r^{n}(1-r),
\end{aligned}
$$

$$
\sum_{n=p}^{\infty}\left|B_{n}-B_{n+1}\right|<\sum_{n=p}^{\infty}\left\{\left|b_{n}-b_{n+1}\right| r^{p}+b_{n+1} r^{n}(1-r)\right\} .
$$

But for $q>p, b_{q}=b_{p}+\sum_{n=p}^{q-1}\left(b_{n+1}-b_{n}\right)$, so $b_{q}=O\left(b_{p}\right)$. Thus

$$
\sum_{n=p}^{\infty}\left|B_{n}-B_{n+1}\right|=O\left(b_{p}\right) r^{p}+O\left(b_{p}\right) r^{p}(1-r)\left(\sum_{n=0}^{\infty} r^{n}\right)=O\left(B_{p}\right) .
$$

Now $\operatorname{Im} f\left(r e^{i \theta}\right)=\sum_{n=1}^{\infty} B_{n} \sin n \theta$ and for each integer $p>0$,

$$
\operatorname{Im} f\left(r e^{i \theta}\right)=\sum_{n=1}^{p} B_{n} \sin n \theta+\sum_{n=p+1}^{\infty}\left(B_{n}-B_{n+1}\right) S_{n}(\theta)-B_{p+1} S_{p}(\theta),
$$

where $S_{n}(\theta)=\sum_{j=1}^{n} \sin j \theta=O(1 / \theta)$. Fix $\theta, 0<\theta \leqq \pi$, let $p$ be such that $\pi /(p+1)<$ $\theta \leqq \pi / p$, and let $1 / 2 \leqq r<1$. Then

$$
\begin{gathered}
\sum_{n=1}^{p}\left|B_{n} \sin n \theta\right|<\sum_{n=1}^{p} B_{n}(n \theta)=\sum_{n=1}^{p} O(1) \theta=p \theta O(1)=O(\pi), \\
\sum_{n=p+1}^{\infty}\left|\left(B_{n}-B_{n+1}\right) S_{n}(\theta)\right|=O\left(B_{p+1}\right) O(1 / \theta)=O(1 /((p+1) \theta))=O(1 / \pi),
\end{gathered}
$$

and

$$
\left|B_{p+1} S_{p}(\theta)\right|=O(1 /((p+1) \theta))=O(1 / \pi) .
$$

Hence $\operatorname{Im} f\left(r e^{i \theta}\right)=O(1)$. Similarly,

$$
\begin{aligned}
\operatorname{Re} f\left(r e^{i \theta}\right) & =\sum_{n=0}^{p} B_{n} \cos n \theta+\sum_{n=p+1}^{\infty} B_{n} \cos n \theta \\
& =\sum_{n=0}^{p} B_{n} \cos n \theta+\sum_{n=p+1}^{\infty}\left(B_{n}-B_{n+1}\right) C_{n}(\theta)-B_{p+1} C_{p}(\theta),
\end{aligned}
$$

where $C_{n}(\theta)=\sum_{j=0}^{n} \cos j \theta=O(1 / \theta)$. Thus, if $b_{n} \geqq 0$ for each $n$, the same argument as above yields $\operatorname{Re} f\left(r e^{i \theta}\right)=\sum_{n=0}^{p} B_{n} \cos n \theta+O(1)$, where the sum is nonnegative if we take $\pi /(2 p+1)<|\theta|<\pi /(2 p)$ and the $O(1)$ is independent of $p$ and $\theta$. Thus we have that $\operatorname{Re} f\left(r e^{i \theta}\right)$ is bounded below for $|\theta| \leqq \pi / 2,1 / 2 \leqq r<1$. But by the first part of Lemma 2 , we have that $\operatorname{Re} f\left(r e^{i \theta}\right)$ is bounded below for $\pi / 2 \leqq$ $|\theta| \leqq 3 \pi / 2,0 \leqq r<1$. Thus, we conclude that $\operatorname{Re} f\left(r e^{i \theta}\right)$ is bounded below for all $\theta$, $0 \leqq \theta \leqq 2 \pi$. This completes the proof of the lemma.

Lemma 3. If $f(z)=\sum a_{n} z^{n}$, where $\left\{a_{n}\right\}$ is a monotone decreasing sequence with $a_{n} \geqq 0$, then $\operatorname{Im} f(z)$ is bounded below for $0 \leqq \arg z \leqq \pi$.

Proof. Fix $r \geqq 0$ and define $h(t)=a_{n} r^{n}$ for $n-1 / 2 \leqq t \leqq n+1 / 2, n \geqq 1, t \geqq 1 / 2$. Then

and

$$
\operatorname{Im} f\left(r e^{i \theta}\right)=\sum_{n=1}^{\infty} a_{n} r^{n} \sin n \theta=(\theta /(2 \sin (\theta / 2))) \int_{1 / 2}^{\infty} h(t) \sin \theta t d t
$$

$$
\int_{1 / 2}^{\infty} h(t) \sin \theta t d t=\int_{1 \mid 2}^{2 \pi / \theta} h(t) \sin \theta t d t+\sum_{n=1}^{\infty} \int_{2 \pi n / \theta}^{2 \pi(n+1) / \theta} h(t) \sin \theta t d t .
$$


The summation term on the right is non-negative, since $h(t)$ is a decreasing function and $\sin \theta t$ is periodic with period $2 \pi / \theta$. Also,

$$
\int_{0}^{1 / 2} a_{1} r \sin \theta t d t+\int_{1 / 2}^{2 \pi / \theta} h(t) \sin \theta t d t \geqq 0
$$

for the same reason. Thus, we have

$$
\operatorname{Im} f\left(r e^{i \theta}\right) \geqq-\int_{0}^{1 / 2} a_{1} r \sin \theta t d t(\theta /(2 \sin (\theta / 2))) \geqq-a_{1} / 2
$$

for $0 \leqq \theta<\pi, r \geqq 0$. This completes the proof.

Lemma 4. If $f(z)=\sum_{n=0}^{\infty} b_{n} z^{2 n+1}$ and if $b_{n} \geqq b_{n+1} \geqq 0$ for each $n \geqq 0$, then $\operatorname{Im} f\left(r e^{i \theta}\right) \geqq 0$ for $0 \leqq \theta \leqq \pi$.

Proof. The argument is the same as in the proof of Lemma 3, except that we let $h(t)=b_{n} r^{2 n+1}$ for $2 n \leqq t<2 n+2$, and $\operatorname{Im} f\left(r e^{i \theta}\right)=(\theta / \sin \theta) \int_{0}^{\infty} h(t) \sin \theta t d t$.

Lemma 5. Let $f(z)$ be defined and meromorphic in $D^{+}=\{z \in D: \operatorname{Im} z>0\}$ such that $f(z)$ has a continuous extension to the interval $(-1,1)$ on the real axis. Let $\operatorname{Im} f(z)$ be bounded below in $D^{+}$and let $f(r) \rightarrow L$ as $r \rightarrow 1-$ (where $L$ is either finite or infinite). For $0<\theta<\pi / 2$, let

$$
D_{\theta}=\left\{z \in D^{+}:-\theta<\arg (1-z)<0\right\} .
$$

Then for each $\theta, 0<\theta<\pi / 2, f(z) \rightarrow L$ as $z \rightarrow 1$ within $D_{\theta}$.

Proof. Let $\Phi: D \rightarrow D^{+}$be a conformal mapping, with $\Phi(1)=1$. Then $\Phi\left(e^{i \theta}\right) \rightarrow 1$ as $\theta \rightarrow 0-$. Consider the function $g(z)=f(\Phi(z))$ for $z \in D$. Then $\operatorname{Im} g(z)$ is bounded below in $D$, so $g(z)$ is a normal function. Further, $g(z)$ has the asymptotic value $L$ at $z=1$. Thus, by a result of Lehto and Virtanen [3, Remark, p. 53], $g(z) \rightarrow L$ in the region $D_{\delta}^{*}=\{z \in D:-\delta<\arg (1-z)\}$ for each choice of $\delta, 0<\delta<\pi / 2$. But this means that $f(z) \rightarrow L$ in each $D_{\theta}$, for given $\theta, 0<\theta<\pi / 2$, there exists a $\delta, 0<$ $\delta<\pi / 2$, such that $D_{\theta} \subset \Phi\left(D_{\delta}^{*}\right)$. This completes the proof of the lemma.

\section{The main results}

We now state and prove our main results.

Theorem 2. If $f(z)=\sum a_{n} z^{n}$ and $g(z)=\sum b_{n} z^{n}$ where $\left\{a_{n}\right\}$ is a decreasing sequence of positive real numbers, and $\left\{b_{n}\right\}$ is a nearly monotone sequence of nonnegative real numbers, and if $h(z)=f(z)+g(z)$, then $h(z)$ is a normal function.

Proof. By Lemma 2, $g(z)$ is bounded near $z=-1$, so $z=e^{i \theta}$ is a normal point for $h(z)$ for $0<\theta<2 \pi$, that is, if $\left\{z_{n}\right\}$ is any sequence of points in $D$ con- 
verging to $e^{i \theta}$, then $\sup \left\{\left(1-\left|z_{n}\right|^{2}\right) h \#\left(z_{n}\right)\right\}<\infty$. Thus, we need only to consider a sequence of points $\left\{z_{n}\right\}$ in $D$ which converges to $z=1$.

By considering a subsequence, if necessary, we note that there are three possibilities to consider: (a) $z_{n} \rightarrow 1$ tangentially, and $\operatorname{Im} z_{n}>0$ for each $n$; (b) $z_{n} \rightarrow 1$ non-tangentially; and (c) $z_{n} \rightarrow 1$ tangentially and $\operatorname{Im} z_{n}<0$ for each $n$. In case (a), we note that both $\operatorname{Im} f\left(z_{n}\right)$ and $\operatorname{Im} g\left(z_{n}\right)$ are bounded below in $D$ by Lemmas 3 and 2, respectively, so that the family $\left\{h_{n}(z)=h\left(\left(z_{n}+z\right) /\left(1+\bar{z}_{n} z\right)\right): z \in D\right\}$ satisfies the condition that $\left\{\operatorname{Im} h_{n}(z)\right\}$ is uniformly bounded below on each compact subset of $D$. It follows that $\left\{h_{n}(z)\right\}$ is a normal family in $D$, so $h_{n}^{\#}(0)=\left(1-\left|z_{n}\right|^{2}\right) h^{\#}\left(z_{n}\right)$ is uniformly bounded. This proves the result in case (a). The proof in case (c) is an easy modification of the proof in case (a), since $\operatorname{Im} h\left(r e^{i \theta}\right)=-\operatorname{Im} h\left(r e^{-i \theta}\right)$. Now, to handle case (b), we use Lemma 5 to conclude that $h(z)$ has the angular limit equal to its radial limit at $z=1$. (We can apply Lemma 5 both to $D^{+}$and to $D^{-}=\left\{z \in D: \bar{z} \in D^{+}\right\}$ where $\operatorname{Im} h(z)$ is bounded above.) It follows that $\left(1-\left|z_{n}\right|^{2}\right) h^{\#}\left(z_{n}\right)$ is bounded in this case also (see [3, Theorem 5, p. 57]). Thus, we conclude that $\left(1-|z|^{2}\right) h \#(z)$ is uniformly bounded in $D$, and the proof is completed.

Theorem 3. If $f(z)=\sum a_{n} z^{2 n+1}$ and $g(z)=\sum b_{n} z^{2 n}$, where $\left\{a_{n}\right\}$ is a monotone decreasing sequence of positive real numbers and $\left\{b_{n}\right\}$ is a nearly monotone sequence of non-negative real numbers such that $b_{n}=O(1 / n)$, and if $h(z)=f(z)+g(z)$ has a radial limit at $z=-1$, then $h(z)$ is a normal function.

The proof of Theorem 3 is essentially the same as the proof of Theorem 2, except that Lemma 4 is used in place of Lemma 3 and the reasoning involving Lemma 5 must be used at both $z=1$ and $z=-1$.

Theorem 4. If $f(z)=\sum a_{n} z^{n}$ and $g(z)=\sum b_{n} z^{n}$, where $\left\{a_{n}\right\}$ is a monotone decreasing sequence of positive real numbers converging to $a \geqq 0, b_{0} \geqq 0$, and $\sum_{n=1}^{\infty}\left|b_{n}-b_{n-1}\right| \leqq b_{0}+a$, then $h(z)=f(z)+g(z)$ is a normal function.

Proof. Since $\quad(1-z) f(z)=a_{0}+\sum_{n=1}^{\infty}\left(a_{n}-a_{n-1}\right) z^{n} \quad$ and $\quad(1-z) g(z)=b_{0}+$ $\sum_{n=1}^{\infty}\left(b_{n}-b_{n-1}\right) z^{n}$, then

$$
h(z)=\left\{a_{0}+b_{0}+\sum_{n=1}^{\infty}\left(\left(a_{n}-a_{n-1}\right) z^{n}+\left(b_{n}-b_{n-1}\right) z^{n}\right)\right\} /(1-z)=d(z) /(1-z) .
$$

Now

$$
\operatorname{Re} d(z) \geqq a_{0}+b_{0}+\left(a-a_{0}\right)-\sum_{n=1}^{\infty}\left|b_{n}-b_{n-1}\right|=b_{0}+a-\sum_{n=1}^{\infty}\left|b_{n}-b_{n-1}\right| \geqq 0 .
$$

Thus, $|\operatorname{Arg} d(z)|<\pi / 2$ and $|\operatorname{Arg}(1-z)|<\pi / 2$ for $z \in D$, so $|\operatorname{Arg} h(z)|<\pi$. Hence $h(z)$ omits all negative values, so $h(z)$ is a normal function. This completes the proof.

Finally, we prove a theorem about analytic functions for which the sequence of coefficients is an increasing sequence. Note that by [1, Example 3, p. 340] the sequence of coefficients can be increasing for a non-normal function. 
Before we can state the theorem, we require another definition. A sequence $\left\{a_{n}\right\}$ of positive real numbers is called naturally convex if there exist two positive numbers $\alpha$ and $\beta$ such that both $0<\alpha \leqq a_{n+1}-a_{n} \leqq \beta<\infty$ and $a_{n}-2 a_{n+1}+a_{n+2} \geqq 0$ for each non-negative integer $n$. Thus, a naturally convex sequence behaves somewhat like the sequence of natural numbers.

Our final theorem is related to [1, Theorem 4, p. 340].

Theorem 5. If $\left\{a_{n}\right\}$ is a naturally convex sequence of positive real numbers, then $f(z)=\sum a_{n} z^{n}$ is a normal function.

Proof. Let $g(z)=(1-z)^{2} f(z)=\sum_{n=0}^{\infty} b_{n} z^{n}$. Then

Let

$$
g(z)=a_{0}+\left(a_{1}-2 a_{0}\right) z+\sum_{n=0}^{\infty}\left(a_{n}-2 a_{n+1}+a_{n+2}\right) z^{n+2} .
$$

$$
g_{n}(z)=a_{0}+\left(a_{1}-2 a_{0}\right) z+\sum_{j=0}^{n-2}\left(a_{j}-2 a_{j+1}+a_{j+2}\right) z^{j+2}, \quad n \geqq 2 .
$$

Note that

$$
\begin{aligned}
\sum_{j=0}^{n}\left|b_{j}\right| & \leqq\left|a_{0}\right|+\left|a_{1}-2 a_{0}\right|+\sum_{j=0}^{n-2}\left(a_{j}-2 a_{j+1}+a_{j+2}\right) \\
& \leqq\left|a_{1}-2 a_{0}\right|+2 a_{0}-a_{1}+a_{n}-a_{n-1} \\
& \leqq 2\left|2 a_{0}-a_{1}\right|+a_{n}-a_{n-1} \\
& \leqq 2\left|2 a_{0}-a_{1}\right|+\beta .
\end{aligned}
$$

Thus we have shown that $\sum\left|b_{n}\right|<\infty$, so $g(z)$ is continuous on the closure of $D$. Further, for $n \geqq 2, g_{n}(1)=a_{n}-a_{n-1} \geqq \alpha>0$. Thus, $g(1) \geqq \alpha>0$. It follows that $f(z)=$ $g(z) /(1-z)^{2}$ can be extended continuously to the boundary of $D$ (with $f(1)=\infty$ ), so $f(z)$ is a normal function.

\section{Some examples}

We give two examples showing that Theorem 5 fails if either aspect of the definition of a naturally convex sequence is missing.

Example 1. Let $g(z)=\exp \left\{1 /(1-z)^{2}\right\}$ and let $f(z)=g(z) /(1-z)^{2}=\sum a_{n} z^{n}$. Then $g(z)$ is not a normal function, but $a_{n}-2 a_{n+1}+a_{n+2} \geqq 0$ and $a_{n}-a_{n-1}>0$ for each $n$.

Proof. Clearly $f(z)$ has the radial limit $\infty$ at $z=1$, but also $f(z) \rightarrow 0$ as $z \rightarrow 1$ along the ray $\operatorname{Arg}(1-z)=3 \pi / 8$. Hence, by a result of Lehto and Virtanen $[3$, Theorem 2, p. 53], $f(z)$ is not a normal function. Now if $g(z)=\sum b_{n} z^{n}$, then

$$
f(z)=\left(\sum b_{n} z^{n}\right)\left(\sum(n+1) z^{n}\right)=\sum\left[(n+1) b_{0}+n b_{1}+\ldots+b_{n}\right] z^{n},
$$


so

$$
\begin{aligned}
& a_{n}=(n+1) b_{0}+n b_{1}+\ldots+b_{n}, \text { and hence } \\
& \qquad a_{n}-a_{n-1}=b_{0}+b_{1}+\ldots+b_{n} \text { and } a_{n}-2 a_{n+1}+a_{n+2}=b_{n+2} .
\end{aligned}
$$

By an easy calculation, $b_{n}>0$ for each $n$, so $f(z)$ satisfies both $a_{n}-a_{n-1}>0$ and $a_{n}-2 a_{n+1}+a_{n+2}>0$.

Example 2. There exists a sequence $\left\{a_{n}\right\}$ of positive real numbers such that $0<\alpha<a_{n+1}-a_{n}<\beta<\infty$, where $\alpha$ and $\beta$ are constants, but $f(z)=\sum a_{n} z^{n}$ is not $a$ normal function.

Proof. By [1, p. 339] there exists a function $g(z)=\sum b_{n} z^{n}$ with $0 \leqq b_{n} \leqq 1 / 2$ and $b_{n} \rightarrow 0$ such that $g(z)$ is not a normal function because of its behavior near $z=i$. If $h(z)=1 /(1-z)^{2}$ and $f(z)=g(z)+h(z)$, then $f(z)=\sum a_{n} z^{n}$, where $a_{n}=$ $n+1+b_{n}$. Thus $f(z)$ is not a normal function but $1 / 2<a_{n+1}-a_{n}<3 / 2$.

We conclude by asking a basic question suggested by our results.

Theorems 2 and 3 raise questions about whether all the hypotheses are necessary. For example, we raise the following question: If $\left\{a_{n}\right\}$ is a monotone decreasing sequence of positive real numbers, and if $\left\{b_{n}\right\}$ satisfies both $b_{n} \geqq 0$ and $b_{n}=O(1 / n)$, is the function $f(z)=\sum\left(a_{n}+b_{n}\right) z^{n}$ a normal function? If the answer is affirmative, then this would greatly simplify Theorem 2 (and probably Theorem 3 also). The condition given in Theorem 2 that $\sum_{n=p}^{\infty}\left|b_{n}-b_{n+1}\right|=O\left(\left|b_{p}\right|\right)$ for each $p$ is a strong restriction on the sequence $\left\{b_{n}\right\}$. It is natural to ask if this can be replaced in the statement of Theorem 2 by some weaker condition.

\section{References}

[1] Lappan, P.: Coefficients and normal functions. - Proc. Amer. Math. Soc. 85, 1982, 335-341.

[2] LAPPAN, P.: Normal functions with bounded coefficients. - Analysis 3, 1983, 305-315.

[3] Lehto, O., and K. I. ViRTANEN: Boundary behaviour and normal meromorphic functions. Acta Math. 97, 1957, 47-65.

[4] Titchmarsh, E. C.: Theory of functions, Second edition. - Oxford University Press, Oxford, 1960.

Academia Sinica

Institute of Mathematics

Taipei, Taiwan

Republic of China
Michigan State University

Department of Mathematics

East Lansing, Michigan 48824

USA

Received 14 January 1986 